\section{Convective convictions}

\section{Stephen Tait}

DOUBLE-diffusive convection is not to be mixed up with double-defective confusion. These different terms for the same phenomenon, the first employed by its proponents and the second by sceptics, may be taken as a light-hearted indication of a lively debate that has been taking place in the geological literature now for more than a decade. The debate concerns the types of convective effects that may occur in molten rock or magma, the stuff of volcanic eruptions, when it is resident in the Earth's crust in magma chambers that are part of subvolcanic plumbing systems. The protractedness of the debate arises not because geologists function as slowly as the processes that they study, but because of the fundamental difficulty of the scientific problem at stake and because it is hard to collect direct evidence to test ideas. On page 390 of this issue, Liang et al.' provide arguably the first convincing observations of doublediffusive convection taking place in melts which, although synthetic, have much in common with naturally occurring magmas.

Natural magmas show a wide range of chemical compositions and, as a result, of physical properties such as viscosity which determine their fluid dynamic behaviour and hence the frequency and type of volcanic eruptions. One of the central preoccupations of igneous petrology has therefore been to try to explain the chemical diversity of magmas. It has been accepted for some time that this variety cannot simply be the result of the melting of different regions of the interior of a heterogeneous Earth, as this would be inconsistent with what is known of the thermodynamics of magmas and would provide a poor and ad hoc explanation of field evidence. Indeed it can be plausibly argued that the principal igneous processes, partial melting and fractional crystallization, have a natural tendency to increase chemical diversity, and it is precisely because much of the Earth's mass has been through the mill of these processes over the aeons of geological time that we find ourselves living on a highly differentiated planet.

Single-component convection takes place in fluids in which one property (most commonly temperature) that can diffuse, and affects the density of a fluid, develops spatial gradients that are acted on by the force of gravity. Double-diffusive, or more generally multicomponent, convection can occur when two or more properties (typically temperature and chemical composition or the concentrations of several different chemical species) are present, if these diffuse at different rates and have opposing effects on the density gradient in the fluid.

Two principal regimes are known that are quite different from single-component flows: layered convection and 'salt-finger' convection. Physical oceanographers are familiar with these from studying the ocean currents generated by gradients of temperature and salinity in the water. Probably the most counter-intuitive property of such flows is that convection can occur even when the overall fluid density decreases vertically upwards, when the gradient would appear to be stable. This happens because the different rates of diffusion of the various components present in the fluid can allow density anomalies to develop and so promote convection. The cooling and crystallization of magma in crustal reservoirs leads to spatial gradients of temperature and of its numerous chemical constituents, and it is now realized that multicomponent convective flows could play a key role in the chemical evolution of magmas. This subject is a nice example of cross-fertilization between scientific disciplines linked by a common physical principle ${ }^{2}$.

The additional twist that Liang et al. give to the story is their claim that not only are different components diffusing at different rates but also off-diagonal terms in the multicomponent diffusion matrix play a crucial role in the redistribution of the species. That is to say, a diffusive flux of one component occurs because of spatial gradients of others. One can think of this phenomenologically as the chemical potential of a component being a function of its local environment because of weak bonding with other components: in short, non-ideality of the solution.

A note of caution may be wise here, as we do not know well what chemical species or complexes are really present in natural silicate melts. The compositions of silicate melts are conventionally expressed as proportions of metallic oxides, a convention followed by Liang et al., but there is no guarantee that these are in fact the thermodynamically relevant components present and diffusing down chemical potential gradients in the melt ${ }^{3}$. A different convention will lead to different off-diagonal terms in the diffusion matrix. However, such off-diagonal terms are expected to arise in the case of a non-ideal solution - that is, when the activities of components are not proportional to their concentrations - and therefore although the numerical magnitudes of the offdiagonal terms will vary with the choice of components, even the most appropriate choice is unlikely to eradicate them.

The approach usually used in extending the ideas of multicomponent convection to geological problems has been to assume that the same fundamental phenomena that can be conveniently observed in

\title{
Taking the beetle by the horns
}

THIS is the business end of the male dung beetle Onthophagus acumina. tus, a native of Central America, which uses its formidable headgear in intraspecific battle. In some beetles in a popula. tion the horns are large, whereas in others they are absent or much reduced in size. Horn size is correlated with the size of the owner, but the trait is discontinuous; that is, male 0 . acuminatus can be divided into two distinct groups according to horn length. Is the explanation for this dimorphism to be found in hereditary or environmental factors? Douglas J. Emlen conducted a series of experiments to find which is the more likely (Proc. $R$. Soc. B256, 131-136; 1994). The particular environmental variable he altered was the amount of food - howler monkey dung - available to beetle larvae, and the answer was clear cut. Horn length is not primarily inherited but is a trait associated with body size and depends on nutrition. This observation, says Emlen, bears on two issues. First, it supports the idea that male secondary sexual characteristics evolve as signals of their possessors' quality. Second, it raises the question of what selective forces maintain the dimorphism. Some genetic 'switch' is probably involved early in a beetle's life, which means the question is as much one for developmental as evolutionary biologists.

T.L. 